MATEC Web of Conferences 21, 02009 (2015)

DOI: $10.1051 /$ matecconf $/ 20152102009$

(C) Owned by the authors, published by EDP Sciences, 2015

\title{
Prediction of grain deformation in drawn copper wire
}

\author{
Chao-Cheng Chang ${ }^{1, \mathrm{a}}$, Zi-Wei Wang ${ }^{1}$, Chien-Kuo Huang ${ }^{2}$, and Hsu-Fu Wu ${ }^{2}$ \\ ${ }^{1}$ Department of Mold and Die Engineering, National Kaohsiung University of Applied Sciences, \\ 415 Chien-Kung Road, Sanmin District, Kaohsiung 80778, Taiwan \\ ${ }^{2}$ Metal Forming Technology Section, Metal Processing R\&D Department, Metal Industries \\ Research \& Development Centre, 1001 Kaonan Highway, Kaohsiung 81160, Taiwan
}

\begin{abstract}
Most copper wire is produced using a drawing process. The crystallographic texture of copper wire, which is strongly associated with grain deformation, can have a profound effect on the formability and mechanical and electrical properties. Thus, the ability to predict grain deformation in drawn copper wire could help to elucidate the evolution of microstructure, which could be highly valuable in product design. This study developed a novel method for predicting grain deformation in drawn copper wire based on finite element simulation with flow net analysis. Simple upsetting tests were conducted to obtain flow stress curves for the simulation of the drawing process. Predictions related to grain deformation were compared with those on the micrographs of the drawn copper wire obtained in experiments. In longitudinal and transverse cross-sections of the drawn wire, the predicted and experiment results presented similar trends involving considerable deformation within the grains. This preliminary study demonstrates the efficacy of the proposed method in providing information useful to the prediction of the grain deformation in drawn copper wire.
\end{abstract}

\section{Introduction}

Copper wire is widely used in telecommunications, power transmission, and other types of electrical equipment. Most copper wire is produced using a drawing process in which plastic deformation leads to changes in grain size and crystallographic texture. Previous studies have shown that inducing grain refinement in copper by applying severe plastic deformation can lead to improvements in mechanical properties [1, 2]. In samples of commercially pure copper processed by equal-channel angular pressing (ECAP), it was shown that electrical conductivity decreases slightly with an increase in the number of passes [3]. That study also demonstrated that yield strength, ultimate tensile stress, and hardness gradually increased with the number of ECAP passes. Moreover, the improvement in mechanical properties and the decrease in electrical conductivity occur in copper wire that has been deformed by the deep wire-drawing process [4], in which the copper underwent severe plastic deformation to form ultrafine microstructures. The angular accumulative drawing process is a recent development aimed

\footnotetext{
${ }^{a}$ Corresponding author: ccchang@kuas.edu.tw
}

This is an Open Access article distributed under the terms of the Creative Commons Attribution License 4.0, which permits unrestricted use, distribution, and reproduction in any medium, provided the original work is properly cited. 


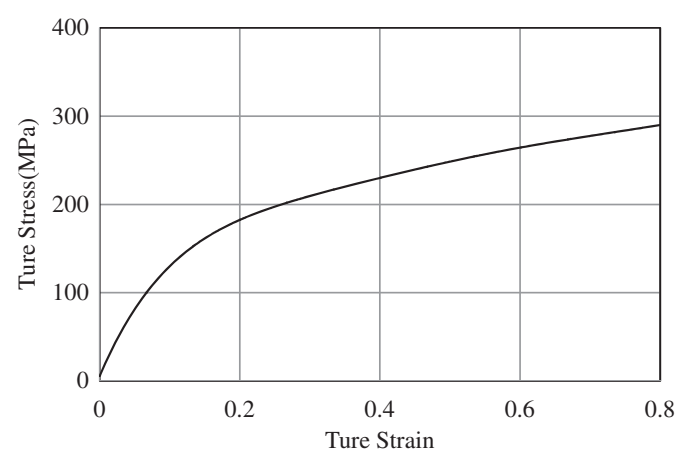

Figure 1. Stress-strain curve.

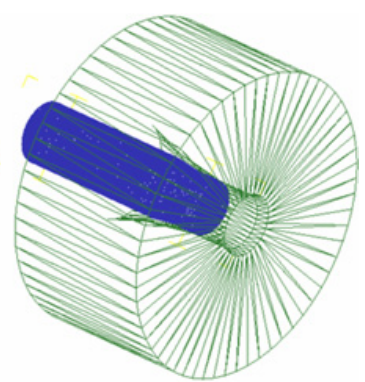

Figure 2. Model of wire drawing process.

at enhancing mechanical properties by using the change in strain path to introduce microstructure inhomogeneity at the cross-section of the wire [5]. The above studies reveal that the crystallographic texture of metal strongly affects the mechanical properties and formability due to its association with grain deformation. Finite-element simulation [6-8] or molecular dynamics simulation [9] can be used to obtain reliable predictions as to the evolution of microstructure in metals. These simulation techniques are able to predict the deformation of grains and thereby elucidate the evolution of microstructure, which can be highly valuable in the subsequent design of products.

This study developed a method based on finite element simulation in conjunction with flow net analysis for the prediction of grain deformation in drawn copper wire. Simple upsetting tests were conducted to obtain the flow stress curves for the simulation of the drawing process. Predictions related to grain deformation in drawn copper were compared with micrograph results obtained from experiments.

\section{Simulation of wire drawing process}

\subsection{Flow stress}

A cast copper rod $8 \mathrm{~mm}$ in diameter was cut into cylindrical specimens $12 \mathrm{~mm}$ in length for use in upsetting tests. Following the application of lubricant to the die surfaces, the specimens were compressed by the die at a speed of $1 \mathrm{~mm}$ per second. The press used in these tests has a capacity of $50 \mathrm{kN}$ load and a position accuracy of $0.01 \mathrm{~mm}$. As shown in Fig. 1, force-displacement data was recorded for use in the calculation of true-stress true-strain curves to simulate the drawing process.

\subsection{Grain deformation and flow net analysis}

The commercial finite element software program DEFORM-3D was used for the simulation of the wire drawing process. The copper wire was drawn at a speed of $1 \mathrm{~mm}$ per second in three stages from an initial diameter of $8 \mathrm{~mm}$ to $7.2 \mathrm{~mm}$, then to $6.4 \mathrm{~mm}$, and finally to a diameter of $5.7 \mathrm{~mm}$. The opening angle of the die was 16 degrees in all three stages of the drawing process. The initial length of the wire was $20 \mathrm{~mm}$. The wire is considered a rigid-plastic material and the dies are considered rigid. The study employed a constant shear model with a friction factor of 0.03 to model the effects of friction at the wire-die interface.

Simulation of grain deformation in the drawing processes was achieved manually recording the grain boundaries in the initial wire from a micrograph using CAD software (AutoCAD) to be imported into DEFORM-3D for flow net analysis. Figure 3 presents micrographs of copper showing patterns in the 

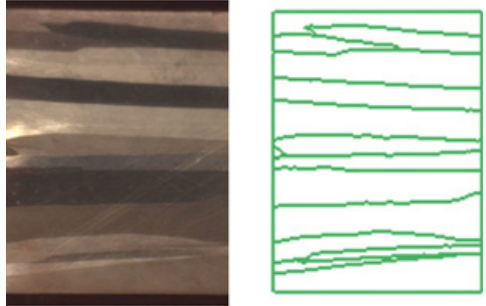

(a) Longitudinal section
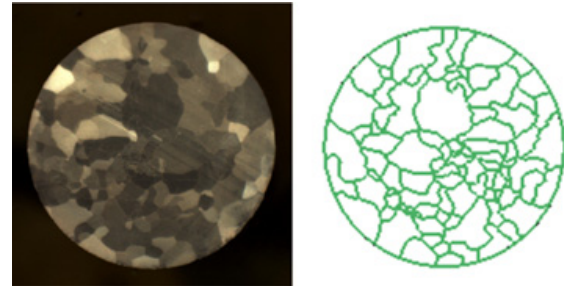

(b) Transverse section

Figure 3. Micrographs of copper showing patterns in grain boundaries.

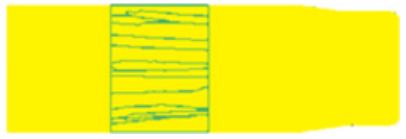

(a) Initial wire

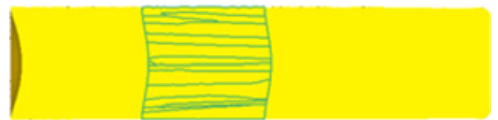

(b) First stage

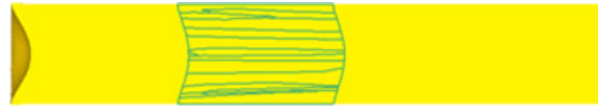

(c) Second stage

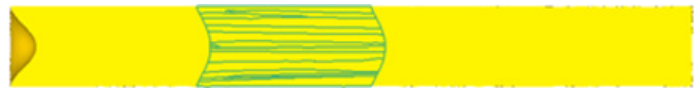

(d) Third stage

Figure 4. Initial and drawn wires with patterns of grain deformations.

grain boundaries. Approximately $200 \mathrm{~K}$ elements were used in the simulations. Predictions related to grain deformation were then compared with the micrographs of the drawn wires.

\section{Results and discussion}

Simulation results revealed that the wire underwent considerable deformation as the diameter was reduced through the three-stage drawing process, as shown in Fig. 4. The patterns, which represent a portion of the grain boundaries in the wire, gradually increased in length (in the longitudinal direction) and reduced in width (in the transverse direction). The shape of the grains became increasingly long and thin. The left end of the drawn wire and the centre of the grain patterns exhibited clear concave surfaces after the first stage of the drawing process.

Figure 5 presents micrographs the deformed grains following the three stages of the drawing process. Micrographs of longitudinal sections clearly illustrate how the grains became increasingly thin as the diameter of the wire was reduced. These results provide evidence of reduced grain size. Similar 


\section{MATEC Web of Conferences}
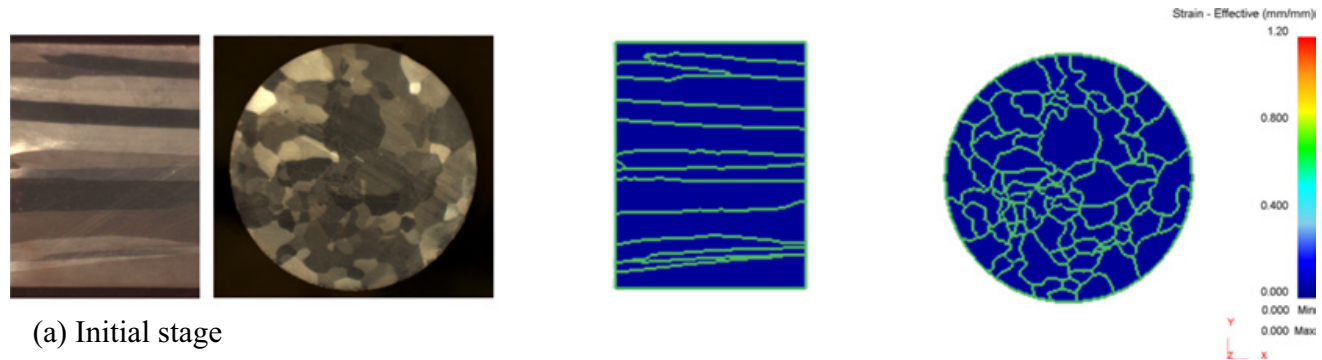

(a) Initial stage
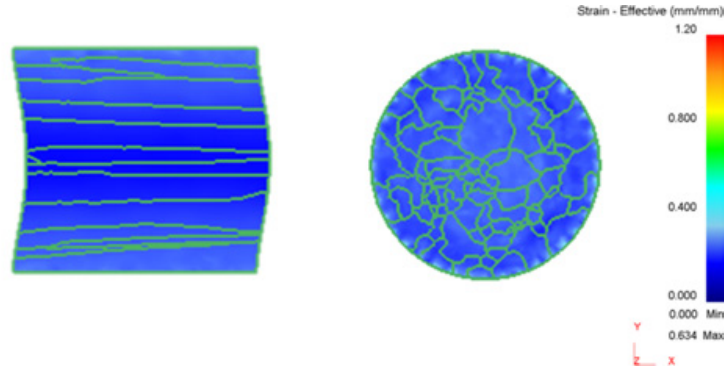

(b) First stage
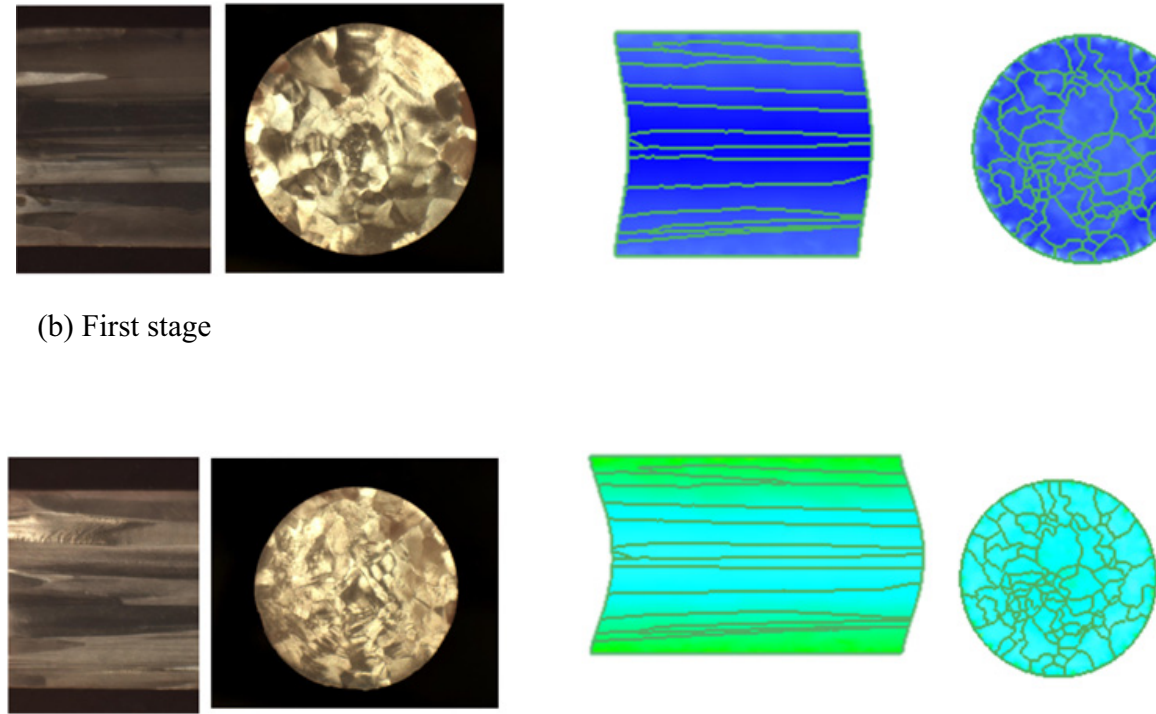

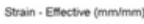
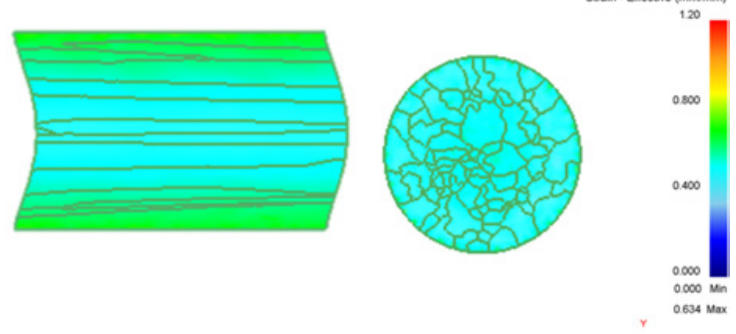

(c) Second stage
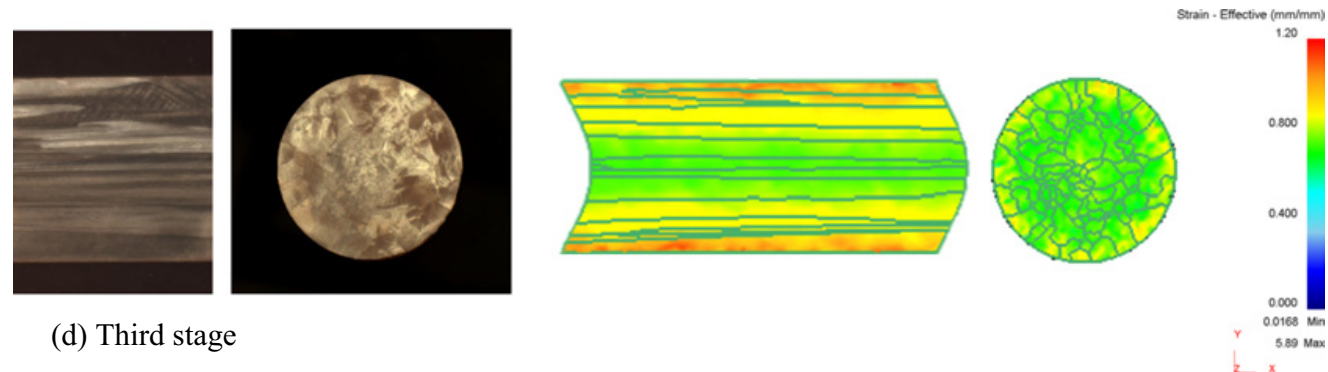

(d) Third stage

Figure 5. Micrographs and effective strain distribution with grain deformation, as predicted by flow net analysis of longitudinal and transverse cross-sections.

phenomena can been observed in transverse sections. Simulation results regarding patterns in the grain boundaries in longitudinal transverse sections also reveal a decrease in grain size with each stage of the drawing process.

Simulation results reveal that the effective strain near the surface of the drawn wire is approximately 1.2 greater than in the centre about 0.6 in the third stage as shown in Fig. 5(d). This is an indication of inhomogeneity with regard to mechanical properties across the transverse section. Further quantitative 


\section{ICNFT 2015}

analysis, including grain size, local strain, and hardness measurements, will be conducted in the near future to further evaluate the proposed method for the prediction of grain deformation.

\section{Summary}

This study used patterns in grain boundaries as the basis for flow net analysis to facilitate finite element simulation of grain deformation in a drawn copper wire. Simulation and experiment results both indicate considerable deformation in the grains as a result of the drawing processes. A decrease in grain size was observed in longitudinal as well as transverse sections of the drawn copper wire. This preliminary study demonstrates the efficacy of finite element simulation in conjunction with flow net analysis in providing reliable predictions related to grain deformation in drawn copper wire. The proposed method is based on patterns in the grain boundaries obtained from actual micrographs of copper; however, numerical methods for the generation of patterns could also be used. The results help to elucidate the evolution of microstructure. This is useful information for product design. The proposed method for the prediction of grain deformation could also be applied to other processes used in the forming of metals.

The authors gratefully acknowledge the financial support from the Ministry of Science and Technology, Taiwan, R.O.C. under Grant No. MOST 103-2221-E-151-014.

\section{References}

[1] R.Z. Valiev, R.K. Islamgaliev, I.V. Alexandrov, Prog. Mater. Sci. 45 (2), 103-189 (2000)

[2] F.H. Dalla Torre, A.A. Gazder, E.V. Pereloma, C.H.J. Davies, J. Mater. Sci. 42 (21), 9097-9111 (2007)

[3] O.F. Higuera-Cobos, J. Cabrera, Mat. Sci. Eng. A-Struct. 571, 103-114 (2013)

[4] K. Hanazaki, N. Shigeiri, N. Tsuji, Mat. Sci. Eng. A-Struct. 527 (21), 5699-5707 (2010)

[5] K. Muszka, L. Madej, J. Majta, Mat. Sci. Eng. A-Struct. 574, 68-74 (2013)

[6] F. Roters, P. Eisenlohr, L. Hantcherli, D. Tjahjanto, T. Bieler, D. Raabe, Acta Mater. 58 (4), $1152-$ $1211(2010)$

[7] P. Van Houtte, A.K. Kanjarla, A. Van Bael, M. Seefeldt, L. Delannay, Eur. J. Mech. A-Solid. 25 (4), 634-648 (2006)

[8] W. Chan, M. Fu, J. Lu, J. Liu, Mat. Sci. Eng. A-Struct. 527 (24), 6638-6648 (2010)

[9] A. Cao, Y. Wei, J. Appl. Phys. 102 (8), 083511 (2007) 